\title{
Research in energy conversion technologies: Policy instruments and uncertainty
}

\author{
Bas Straathof ${ }^{1}$ \\ Adriaan van Zon ${ }^{2}$
}

January, 2002

\begin{abstract}
:
In this paper, the effects of uncertainty and of various policy instruments on the length and attractiveness of private research projects are studied. Research expenditure can be regained from quasi-rents that are earned by exploiting patents on the fruits of research. The accumulation of knowledge is modeled as a Poisson process. Within the context of the model, we show that firms shorten the duration of research projects when uncertainty in knowledge accumulation increases or when, for example, the validity of patents is prolonged. The underlying mechanisms are due to Jensen's Inequality and a real-option effect. Furthermore, we develop a smooth pasting condition for a class of Poisson processes.
\end{abstract}

Keywords: R\&D, Energy conversion, Real-option theory, Poisson process

\footnotetext{
${ }^{1}$ MERIT - Maastricht University, P.O. Box 616 - 6200 MD Maastricht - The Netherlands, tel. +31 43 3883879, b.straathof@merit.unimaas.nl.

${ }^{2}$ MERIT - Maastricht University.
} 


\section{Introduction}

The presence of uncertainty has an impact on the technology that is used for energy conversion. Uncertainty changes the expenditure on research that is done by firms. Two remarks can be made here. First, uncertainty may change the expected value of a research project. Second, uncertainty may cause firms to postpone shutting down a research project. According to the literature on real options (e.g. Dixit and Pindyck, 1994), the presence of uncertainty gives a value to the option to postpone an irreversible investment. As the development costs of a newly discovered technology are irreversible, the timing of stopping research and starting the development of a technology becomes important. Goel and Ram (2001) find empirical evidence that real-option effects are important for R\&D expenditure.

Section two discusses the distinction between basic and applied research. Some remarks on the effects of competition on research can be found in section three. In section four, a simple formal example is given of how the intrinsic uncertainty of research can affect the implementation of a new technology. In this example, an irreversible investment is made based on the technology that results from a research project. The research project is stopped at the moment that the investment project is started. The fifth section treats the effects of various policy instruments on the way firms conduct research. Concluding remarks and a brief outline for further research can be found in the sixth section.

\section{The intrinsic uncertainty of research}

Research is about discovering the unknown. By its nature, its results are uncertain. This does not mean that all research is equally uncertain, of course. A distinction can be made between research projects with a high degree of uncertainty and research projects with a low degree of uncertainty. The outcome of a project is generally more certain when there is a clear idea about what this outcome should look like. For example, there is not much uncertainty involved when a car manufacturer wants to develop a small, safe, and luxurious car. Although the costs of developing this car are not completely certain, it is highly probable that the manufacturer will succeed in developing the car. Of course, whether it is able to sell the car is a different question.

When the objective of the project is less clear, it is also less clear whether the objective will be realized, and, equivalently, what its costs will be. As an example, consider the development of an environmentally safe energy conversion method that generates cheap electricity. The road from the simple idea of wind energy to its large scale implementation has been quite long, and it is still uncertain if wind energy can be made a cheap source of energy compared to, for example, nuclear energy. ${ }^{3}$ This is even truer for the idea of solar energy, and the idea of nuclear fusion as a source of energy.

When the objective of a project can be specified in detail, the research done is aimed at the application of more general knowledge that is readily available. Therefore, it is called

\footnotetext{
${ }^{3}$ Renewing Faith, The Economist, February $10^{\text {th }}, 2001$
} 
applied research. When this more general knowledge is not available, it is not possible to specify the objective of a project in great detail. Research aimed at acquiring general knowledge is called basic research. In the absence of more general knowledge, a project's objective cannot be achieved without basic research. There is no point in applying something unknown.

Is the outcome of basic research more uncertain than that of applied research? It has to be by definition: Applied research assumes the existence of more general knowledge, while basic research assumes the absence of more general knowledge. As less knowledge means more uncertainty, basic research is more uncertain than applied research. Of course, the concepts of basic and applied research are relative. After all, research on energy from nuclear fusion is for a large part an application of theories from physics.

\section{Competition and research}

Suppose a firm is not alone in doing research in a particular field. Other firms or universities could be doing similar research. In this case, the knowledge obtained by research may become less valuable to an individual firm because several firms have access to it. Consequently, the firm cannot engage in monopoly pricing. Even worse, there could be a possibility that one firm develops a technology that is superior to the technologies other firms are working on. If this happens, the research done by the others becomes worthless altogether. ${ }^{4}$ The same thing happens when one firm is rewarded a patent for its research efforts because it was the first to obtain a patentable result. The research done by other firms becomes useless because it is illegal for them to use their knowledge. As a result, firms may find themselves in the midst of a patent race, in which the winner takes all. A patent races leads to high uncertainty in the value of the outcome of a research project

When a firm succeeds in obtaining a monopoly on a body of knowledge, this gives the firm an advantage over its competitors. Successful research is thus expected to result in (quasi-)imperfect competition. Does this mean that firms necessarily make real profits? No. When an incumbent firm is able to make high profits other firms will also do research in order to enter the market. Therefore, the discounted profits from the exclusive possession of knowledge should be expected to just make up for the discounted expenditure on research. When every firm is equally capable to do research, firms can only obtain quasi-rents from their research. Only firms that have an advantage in researching are likely to make monopoly profits.

However, even if the firm has an advantage in researching, competition may cause uncertainty. When results from research done by a particular firm are easily obtained and used by competitors, this will set the firm that has spend resources on research at a disadvantage. As a result, firms that are unable to protect the outcome of their research

\footnotetext{
${ }^{4}$ When this knowledge provides a starting point for further research, which is quite common, it may still be valuable to the firm.
} 
will not do research. ${ }^{5,6}$ This phenomenon, called business stealing, has not only a substantial depressing effect on the pace of innovation, it also brings the element of uncertainty into decisions on research expenditure.

Competition makes the size of monopoly rents on the outcome of research uncertain. Two causes of this uncertainty can be pointed out. Patent races make highly uncertain which of the researching firms gets the reward for its research, while business stealing reduces this reward for all firms that do research. The phenomenon that a new technology replaces the current technology has been studied first by Joseph Schumpeter (1934). He called this phenomenon creative destruction. An important contribution in the context of a growth model has been made by Aghion and Howitt (1992).

\section{$4 \quad$ Research expenditure and investment timing}

In this section, a formal example is given of how uncertainty affects the length of a research project. First, an introduction to the context of the model is given. Second, the model is presented.

\subsection{Introduction to the model}

When it comes to research into new energy conversion technologies, two types of decisions are important. First, a firm has to decide how much and when to invest in research. This is an important issue because expenditure on research can never be reversed. Second, it has to decide when to exploit the results of the research, that is, when a new technology should be implemented. Because implementation of a new technology is considered here to be an irreversible investment project, the timing of implementation matters. These two types of decisions are related to each other. One can only decide how much to spend on research when one has an idea about the value of the investment project. The value of the investment project, in turn, depends on the amount of money that has been spent on research.

You are never certain how costly it will be to achieve the objective of the research. This intrinsic uncertainty typically is a form of individual uncertainty as every firm keeps the results of research to itself. Intrinsic uncertainty is about how much knowledge is generated by doing research. For a given research period, the knowledge of the firm is expected to increase on several occasions. Therefore, intrinsic uncertainty can best be modeled as a jump process.

In the model, research expenditure per unit of time is fixed. Total research expenditure can only be influenced by stopping the research project. At the same moment that the research project is stopped, the technology is further developed to make it usable in practice, and a patent is granted. The model thus only treats one decision. Furthermore, only one type of uncertainty is considered: the intrinsic uncertainty of research, which is modeled as a Poisson process.

\footnotetext{
${ }^{5}$ An exception is, of course, when the firm gets paid for doing research as were it a service. For example, the government might pay a firm to do research in a field were no exclusive control can exist over the output of the research.

${ }^{6}$ Romer (1990) discusses the concept of excludability in more detail.
} 


\subsection{The model}

This sub-section gives an illustration of the impact of uncertainty on the length of a simple research project. The methodology followed here is similar to that of Dixit and Pindyck (1994).

Consider an economy with only one type of final product that is produced with use of labor and energy. Energy is converted by plants that don't use variable inputs and are built with capital. Each generation of plants is assumed to be of a different technology. The amount of capital needed is proportional to the 'raw' capacity of a generation of plants.

$$
\begin{aligned}
Y & =L^{1-\alpha} U^{\alpha} \\
U & =\left(\sum_{n} x_{i}^{\beta}\right)^{\frac{1}{\beta}} \\
K & =\sum_{n} q^{\eta_{i}} x_{i}
\end{aligned}
$$

Here, $x_{i}$ is the capacity for energy conversion of plants belonging to generation $i \in n, U$ the effective total capacity for energy conversion, $K$ the total amount of capital, and $Y$ the amount of final production. The parameter $\eta_{i}$ stands for the amount of knowledge embodied in the capacity. The parameter $q<1$ determines by how much an increase in knowledge intensity will reduce the capital intensity of the technology. Technologies are assumed to be imperfect substitutes. Therefore, effective capacity is a CES aggregate of the different types of capacity. Imperfect substitutability is assumed to be appropriate for two reasons. First, governments often require that several technologies are simultaneously used in order to diversify the risks that are associated with specific technologies. Second, technological heterogeneity induces imperfect substitutability. For example, some technologies may have a geographical advantage over other technologies because of the local availability of energy sources (e.g. wind or sun). Also, the responsiveness of plants to react to changes in demand (e.g. peak hours) differs per technology.

Research and development is done by private $R \& D$ firms alone. An R\&D firm gets a patent for each technology it invents and develops. The patent expires after $\tau$ years. After a patent has been obtained, the firm can sell the patent or it can license the technology to firms that build and maintain plants. In either case, the attractiveness of doing research depends on the stream profits a patent is going to generate.

Profits appropriated by the owner of a patent are given by

$$
\pi_{i}=p_{i} x_{i}-r q^{\eta_{i}} x_{i}
$$

The supply and inverted demand functions with regard to capacity of type $i$ are 


$$
\begin{aligned}
& \bar{p}_{i}=\frac{1}{\beta} r q^{\eta_{i}} \\
& p_{i}=\alpha L^{1-\alpha}\left(\sum_{n} x_{i}^{\beta}\right)^{\frac{\alpha}{\beta}-1} x_{i}^{\beta-1}
\end{aligned}
$$

respectively. ${ }^{7}$ Equating both expressions in (4.3) gives the optimal capacity of that is to be installed of technology $i$

$$
x_{i}=(\alpha \beta)^{\frac{1}{1-\beta}} r^{\frac{1}{\beta-1}} q^{\frac{\eta_{i}}{\beta-1}} L^{\frac{\alpha-1}{\beta-1}}\left(\sum_{n} x_{i}^{\beta}\right)^{\frac{\beta-\alpha}{\beta(\beta-1)}}
$$

Profits can be derived by using equations (4.3), (4.4) and (4.2)

$$
\begin{aligned}
\pi_{i} & =(1-\beta) \bar{p}_{i} \bar{x}_{i} \\
& =(1-\beta) \alpha^{\frac{1}{1-\beta}} \beta^{\frac{\beta}{1-\beta}} r^{\frac{\beta}{\beta-1}} q^{\frac{\beta \eta_{i}}{\beta-1}} L^{\frac{\alpha-1}{\beta-1}} U^{\frac{\beta-\alpha}{\beta-1}}
\end{aligned}
$$

The profitability of a technology depends on its knowledge intensity on and, if $\alpha \neq \beta$, on the total effective capacity installed. For simplicity, we assume that changes in total effective capacity are regarded to be exogenous by R\&D firms. In particular, R\&D firms use the following rule of thumb

$$
U_{t}=U_{0} \exp [v t]
$$

As a patent expires after $\tau$ years, the value of a new patent is

$$
\begin{aligned}
V_{i} & =\int_{0}^{\tau} \pi_{i t} e^{-r t} \mathrm{~d} t \\
& =\int_{0}^{\tau} \pi_{i 0} \exp \left[\left(\frac{\beta-\alpha}{\beta-1} v-r\right) t\right] \mathrm{d} t \\
& =\Gamma_{0} \exp \left[\ln q \frac{\beta}{\beta-1} \eta_{i}\right] \\
\Gamma_{0} & =(1-\beta) \alpha^{\frac{1}{1-\beta}} \beta^{\frac{\beta}{1-\beta}} r^{\frac{\beta}{\beta-1}} L^{\frac{\alpha-1}{\beta-1}} U_{0}^{\frac{\beta-\alpha}{\beta-1}} \frac{1-\exp \left[\left(\frac{\beta-\alpha}{\beta-1} v-r\right) \tau\right]}{r-\frac{\beta-\alpha}{\beta-1} v}
\end{aligned}
$$

By doing research, the capital intensity of energy conversion capacity can be lowered. The amount of capital needed for new unit of capacity is given by $q^{\eta_{i}}$. Doing research is assumed to cost a fixed amount per unit of time $(\varepsilon)$ and is expected to generate the following results:

\footnotetext{
${ }^{7}$ The supply price is derived using the Amoroso-Robinson condition: $p_{i}=\varepsilon_{x p} /\left(1+\varepsilon_{x p}\right) r q^{\eta_{i}}$, with $\varepsilon_{x p}=(\mathrm{d} x / x) /(\mathrm{d} p / p)$. The inverted demand function is derived by equating the price with the marginal product of $x_{i}$.
} 


$$
\begin{aligned}
\Delta \eta_{i} & =u \text { with probability } \lambda \mathrm{d} t \\
\Delta \eta_{i} & =\varphi u \text { with probability }(1-\lambda) \mathrm{d} t \\
0 & \leq \lambda \leq 1, \quad u>0
\end{aligned}
$$

An increase in knowledge $(\eta)$ implies a decrease in the capital intensity of future capacity. The parameter $\varphi$ assigns a weight to normal and exceptional changes. The expected change in $\eta$ is given by

$$
\langle\Delta \eta\rangle=\varphi u \mathrm{~d} t+(1-\varphi) u \lambda \mathrm{d} t
$$

Both $\lambda=0$ and $\varphi=1$ represent deterministic change. For

$$
\varphi=\frac{\lambda}{\lambda-1}
$$

the expected change in knowledge is zero.

Before the a plant can be built, the fruits of research have to be made ready to implement by means of a development project. The development project is assumed to cost a fixed amount $D$. Also, it is assumed not to take any time. According to Bellman's principle of optimality, the following condition has to be satisfied

$$
r R=-\varepsilon+\frac{\langle\mathrm{d} R\rangle}{\mathrm{d} t}
$$

With

$$
\begin{aligned}
\frac{\langle\Delta R\rangle}{\mathrm{d} t} & =[R(V(\eta))-R(V(\eta-(1-\varphi) u))] \lambda+\frac{\partial R}{\partial V}\left(\left.\frac{\partial V}{\partial t}\right|_{\text {no jump }}\right) \\
& =[R(V(\eta))-R(V(\eta-(1-\varphi) u))] \lambda+\frac{\partial R}{\partial V} \ln q_{\frac{\beta}{\beta-1}} \varphi u V(\eta)
\end{aligned}
$$

Besides the Bellman equation (4.11), two other conditions have to be satisfied for the determination of the value of the research project. First, the value of the research project at the time it is optimal to start the investment project should be equal to the value of the plant minus the development costs. That is,

$$
R\left(V^{*}\right)=V^{*}-D
$$

This condition is called the value matching condition. Second, the increase in the value of the research project at the time the optimal value of the plant is reached should be equal to the increase in the value of the plant. This has to apply when the optimal plant value is reached by a jump in $\eta$ or not. $^{8}$

\footnotetext{
${ }^{8}$ It is assumed that a jump in $\eta$ will not lead to $V>V^{*}$.
} 


$$
\begin{gathered}
\left.\frac{\partial R\left(V^{*}\right)}{\partial V^{*}}\right|_{\text {no jump }}=1 \\
\left.\Delta R\right|_{\text {jump }}=\left.\Delta V\right|_{\text {jump }} \\
R(V(\eta))-R(V(\eta-(1-\varphi) u))+\frac{\partial R}{\partial V} \ln q \frac{\beta}{\beta-1} \varphi u V(\eta) \\
= \\
V(\eta)-V(\eta-(1-\varphi) u)+\ln q \frac{\beta}{\beta-1} \varphi u V(\eta),
\end{gathered}
$$

and thus

$$
R(V(\eta))-R(V(\eta-(1-\varphi) u))=V(\eta)-V(\eta-(1-\varphi) u)
$$

From (4.14) and (4.16) follows that also the expected changes in $R$ and $V$ are equal.

$$
\left.\frac{\langle\Delta R\rangle}{\mathrm{d} t}\right|_{\eta=\eta^{*}}=\left.\frac{\langle\Delta V\rangle}{\mathrm{d} t}\right|_{\eta=\eta^{*}}
$$

This condition, called the smooth pasting condition, is necessary because if otherwise, postponing development at the optimal time would have a larger expected value than the value of the plant, which would contradict that development at that time is optimal.

Using equations (4.13), (4.14), and (4.16) into equation (4.12) gives

$$
\begin{aligned}
\frac{\langle\Delta R\rangle}{\mathrm{d} t} & =\left[V^{*}-V\left(\eta^{*}-(1-\varphi) u\right)\right] \lambda+\ln q \frac{\beta}{\beta-1} \varphi u V^{*} \\
& =\Omega V^{*} \\
\Omega & =\lambda\left(1-\exp \left[-\ln q \frac{\beta}{\beta-1}(1-\varphi) u\right]\right)+\ln q \frac{\beta}{\beta-1} \varphi u
\end{aligned}
$$

Inserting (4.18) into (4.11) yields

$$
r\left(V^{*}-D\right)=-\varepsilon+\Omega V^{*}
$$

The value of the plant at which its development is optimal is

$$
V^{*}=\frac{r D-\varepsilon}{r-\Omega}
$$

$V$ can only be positive if $r D_{>}^{<} \varepsilon$ and $r_{>}^{<} \Omega$. The expected time $\left\langle\Delta^{*} t\right\rangle$ it is going to take to reach $V^{*}$ from $V_{t}$ can be derived as follows 


$$
\begin{gathered}
V^{*}-V_{t}=\Gamma_{t+\Delta^{*} t} \exp \left[\ln q \frac{\beta}{\beta-1}\left(\eta_{t}+\Delta^{*} \eta\right)\right]-\Gamma_{t} \exp \left[\ln q \frac{\beta}{\beta-1} \eta_{t}\right] \\
=V_{t}\left(\exp \left[\left(\ln q \frac{\beta}{\beta-1}[(1-\varphi) \lambda+\varphi] u+\frac{\beta-\alpha}{\beta-1} v\right)\left\langle\Delta^{*} t\right\rangle\right]-1\right) \\
\left\langle\Delta^{*} t\right\rangle=\frac{1}{\ln q \frac{\beta}{\beta-1}[(1-\varphi) \lambda+\varphi] u+\frac{\beta-\alpha}{\beta-1} v} \ln \left(\frac{V^{*}}{V_{t}}\right) \\
=\frac{1}{\ln q \frac{\beta}{\beta-1}[(1-\varphi) \lambda+\varphi] u+\frac{\beta-\alpha}{\beta-1} v} \ln \left(\frac{r D-\varepsilon}{[r-\Omega] \Gamma_{t} \exp \left[\ln q \frac{\beta}{\beta-1} \eta_{t}\right]}\right)
\end{gathered}
$$

Next, we are going to derive the value of the research project $R$ as function of $V$. The function $R(V)$ has to satisfy conditions $(4.13)-(4.17)$ together with $R(0)=0 .{ }^{9} \mathrm{~A}$ functional form that meets these conditions is

$$
R(V)=B V^{b}
$$

Conditions (4.13), (4.14), and (4.20) can be used to solve for $b$ and $B$.

$$
\begin{gathered}
\left.\begin{array}{c}
B V^{* b}=V^{*}-D \\
b B V^{* b-1}=1
\end{array}\right\} V^{*}=\frac{b}{b-1} D \\
b=\frac{r D-\varepsilon}{\Omega D-\varepsilon} \\
B=\frac{(b-1)^{b-1}}{b^{b} D^{b-1}}
\end{gathered}
$$

As $r D_{>}^{<} \varepsilon$ for $r<\Omega$ we have $b>1$. This assures that $R(V) \geq V-D$.

\subsection{Comparing certainty and uncertainty}

In order to be able to change the level of uncertainty while keeping the expected value of $\eta$ constant, we set $\varphi$ according to (4.10). We can assess the effects of mean-preserving changes in uncertainty by varying $\lambda$. Inserting equation (4.10) into $\Omega$ makes

$$
\Omega^{\prime}=\lambda\left(1-\exp \left[\ln q \frac{\beta}{\beta-1} \frac{1}{1-\lambda} u\right]\right)+\ln q \frac{\beta}{\beta-1} \frac{\lambda}{\lambda-1} u .
$$

The value of the project without uncertainty is

\footnotetext{
${ }^{9} V=0$ implies $\eta \rightarrow \infty$. Therefore, no finite decrease in $\eta$ can make the value of the plant become positive.
} 


$$
\left.V^{*}\right|_{\lambda=0}=D-\frac{\varepsilon}{r}
$$

The effect of a marginal change in uncertainty is

$$
\frac{d V^{*}}{d \lambda}=\frac{r D-\varepsilon}{\left(r-\Omega^{\prime}\right)^{2}}\left(\begin{array}{l}
1-\exp \left[\ln q \frac{\beta}{\beta-1} u \frac{1}{1-\lambda}\right]-\exp \left[\ln q \frac{\beta}{\beta-1} \frac{1}{1-\lambda} u\right] \ln q \frac{\beta}{\beta-1} u \frac{\lambda}{(\lambda-1)^{2}} \\
+\ln q \frac{\beta}{\beta-1} u\left(\frac{1}{\lambda-1}-\frac{\lambda}{(\lambda-1)^{2}}\right)
\end{array}\right) .
$$

For $r D>(<) \varepsilon$ uncertainty affects the stopping value of a research project negatively (positively).

The expected time before development is

$$
\begin{aligned}
\left\langle\Delta^{*} t\right\rangle & =\frac{1}{\ln q \frac{\beta}{\beta-1} \frac{\lambda}{\lambda-1} u+\frac{\beta-\alpha}{\beta-1} v} \ln \left(\frac{V^{*}}{V_{t}}\right) \\
& =\frac{1}{\ln q \frac{\beta}{\beta-1} \frac{\lambda}{\lambda-1} u+\frac{\beta-\alpha}{\beta-1} v} \ln \left(\frac{r D-\varepsilon}{\left[r-\Omega^{\prime}\right] \Gamma_{t} \exp \left[\ln q \frac{\beta}{\beta-1} \eta_{t}\right]}\right)
\end{aligned}
$$

More uncertainty induces $\mathrm{R} \& \mathrm{D}$ firms to shorten their research projects (ruling out $V<0$ and $V^{*}<V$ ). In the absence of uncertainty development is started immediately as the value of the patent does not change 


\section{Policy instruments}

\section{Subsidy on research expenditure $(\varepsilon)$}

From equation (4.22) it is clear that a reduction of $\varepsilon$ will cause the length of the research project to increase. This has two important implications. First, the capital intensity of new energy conversion capacity will be higher. Second, it will take longer before new technology is introduced. If the research is subsidized because of its environmental desirability, subsidizing research expenditure will be counterproductive.

\section{Subsidy on development expenditure $(D)$}

From equation (4.22) it is clear that a reduction of $D$ will cause the length of the research project to decrease. The effects of a subsidy on development expenditure opposite to the effects of a subsidy on research expenditure.

Subsidy on capacity $(\boldsymbol{x})$

Introduce a subsidy $(g)$ on capacity in equation (4.5)

$$
\pi=(\alpha+g) \overline{p x}
$$

The subsidy will increase the current value of the plant while leaving the optimal value of the plant unaffected. As a result development of the plant will be started earlier and the capital intensity of the plant will be higher (equation (4.22)).

All subsidies will increase the value of research projects $(R)$. Subsidies will make research projects feasible that were previously unattractive. Subsidies will increase the variety of directions in which research is done thereby reducing the risk of having to use capital-intensive capacity for a long time.

\section{Make patents last longer $(\tau)$}

The current value of a patent is raised by lengthening the period of its validity as can be seen from equation (4.7). From (4.22) follows that increasing the duration of patents shortens the research project as the current value of a patent now lies closer to the stopping value then before. Making patents last longer increases the incentives for starting research but decreases the length of research projects.

\section{Stimulate competition in the energy market $(\boldsymbol{\beta})$}

Equations (4.20) and (4.26) show that stimulating competition in the energy market by letting $\beta$ approach one, reduces the value of a patent as quasi-rents diminish. 


\section{Concluding remarks}

The model presented in section three illustrates that the intrinsic uncertainty of research partly determines the technology on which investments are based. In the context of the model, the impact of uncertainty depends on the magnitude of the variables. In specific cases, less money will be spent on applied (low-risk) research than on (high-risk) basic research. Policy instruments may increase the attractiveness for firms to do research but it depends on the instrument whether this will take the form of a few new but longer projects or many shorter projects.

Decisions on the expenditure on research and the timing of investments are usually more complicated than the one described by the model. The model can be extended in several ways. For instance, uncertainty in the value of existing plants can be introduced. This uncertainty could be modeled to be caused by uncertainty in demand, supply, the effects of competition, interest rates, or environmental policies. Also, the expenditure on research per unit of time could be made endogenous by letting it affect the probability or size of a jump. The model can be extended to account for general equilibrium. Furthermore, research may include not just one but several dimensions, such as quality, efficient use of inputs, environmental safety, or flexibility in output levels. 


\section{References}

Aghion, Philippe and Howitt, Peter. "A Model of Growth through Creative Destruction." Econometrica, 1992, 60(2), pp. 323-51. . Endogenous Growth Theory. Cambridge and London: MIT Press, 1998.

Barro, Robert J. and Sala-i-Martin, Xavier. Economic Growth. New York: McGrawHill, 1995.

Dixit, Avinash K. and Pindyck, Robert S. Investment under Uncertainty. Princeton: Princeton University Press, 1994.

The Economist. "Renewing Faith," February 10th, 2001a, Survey Energy 8.

. "The Slumbering Giants Awake," February 10th, 2001b, Survey Energy 6-11.

Goel, Rajeev K. and Ram, Rati. "Irreversibility of R\&D Investment and the Adverse Effect of Uncertainty: Evidence from the OECD Countries." Economics Letters, 2001, 71(2), pp. 287-91.

Romer, Paul M. "Endogenous Technological Change." Journal of Political Economy, 1990, 98(5), pp. S71-S102.

Schumpeter, Joseph A. The Theory of Economic Development. Cambridge MA: Harvard University Press, 1934. 